\title{
Limited Evidence for Risk Factors for Proarrhythmia and Sudden Cardiac Death in Patients Using Antidepressants: Dutch Consensus on ECG Monitoring
}

\author{
Mirjam Simoons $^{1,2,3}$ (D) Adrie Seldenrijk ${ }^{2,4} \cdot$ Hans Mulder $^{1,5} \cdot$ Tom Birkenhäger $^{6,7} \cdot$ \\ Mascha Groothedde-Kuyvenhoven ${ }^{8} \cdot \operatorname{Rob~Kok}^{9} \cdot$ Cornelis Kramers $^{10}$. \\ Wim Verbeeck ${ }^{11,12}$ - Mirjam Westra' ${ }^{2}$ Eric van Roon ${ }^{3,13}$ - Roberto Bakker ${ }^{14,15}$. \\ Henricus Ruhé ${ }^{2,16,17}$
}

Published online: 26 February 2018

(C) The Author(s) 2018. This article is an open access publication

\begin{abstract}
Currently, there is a lack of international and national guidelines or consensus documents with specific recommendations for electrocardiogram (ECG) screening and monitoring during antidepressant treatment. To make a proper estimation of the risk of cardiac arrhythmias and sudden (cardiac) death during antidepressant use, both the drug and patient-specific factors should be taken into account; however, solid evidence on how this should be done in clinical practice is lacking. Available
\end{abstract}

Mirjam Simoons and Adrie Seldenrijk share first authorship.

Roberto Bakker and Henricus Ruhé share senior authorship.

Electronic supplementary material The online version of this article (https://doi.org/10.1007/s40264-018-0649-z) contains supplementary material, which is available to authorized users.

Henricus Ruhé

h.g.ruhe@umcg.nl

1 Department of Clinical Pharmacy, Wilhelmina Hospital Assen, Assen, The Netherlands

2 Department of Psychiatry, Interdisciplinary Centre for Psychopathology and Emotion Regulation, University Medical Centre Groningen, University of Groningen, Groningen, The Netherlands

3 Unit of Pharmacotherapy, -Epidemiology and -Economics, Department of Pharmacy, University of Groningen, Groningen, The Netherlands

4 Department of Research and Innovation, GGZ InGeest, Amsterdam, The Netherlands

5 Psychiatric Hospital GGZ Drenthe, Assen, The Netherlands

6 Department of Psychiatry, Erasmus Medical Centre, Rotterdam, The Netherlands

7 Collaborative Antwerp Psychiatric Research Institute (CAPRI), University of Antwerp, Antwerp, Belgium recommendations on the management of QT(c) prolongation (with antidepressant treatment) emphasize that special attention should be given to high-risk patients; however, clinicians are in need of more concrete suggestions about how to select patients for ECG screening and monitoring. Based on a review of the literature, a Dutch multidisciplinary expert panel aimed to formulate specific guidelines to identify patients at risk for cardiac arrhythmias and sudden death by developing a consensus statement regarding ECG screening before, and monitoring during, antidepressant use. We first reviewed the literature to identify the relative risks of various risk factors on cardiac arrhythmia and sudden (cardiac) death during antidepressant use. These relative contributions of risk factors could not be determined since no systematic reviews or metaanalyses quantitatively addressed this topic. Because

8 Department of Clinical Pharmacy, Deventer Hospital, Deventer, The Netherlands

9 Department of Old Age Psychiatry, Parnassia Psychiatric Institute, The Hague, The Netherlands

10 Department of Internal Medicine, Radboudumc, Nijmegen, The Netherlands

11 Vincent van Gogh Institute for Psychiatry, ADHD and Autism Circuit, Venray, The Netherlands

12 Department of Pharmacology and Toxicology, Radboudumc, Nijmegen, The Netherlands

13 Department of Clinical Pharmacy and Clinical Pharmacology, Medical Centre Leeuwarden, Leeuwarden, The Netherlands

14 Psychiatric Centre GGz Centraal, Amersfoort, The Netherlands

15 Department of Psychiatry and Psychology, South Limburg Mental Health and Teaching Network, Maastricht University Medical Centre, Maastricht, The Netherlands 
evidence was insufficient, additional expert opinion was used to formulate recommendations. This resulted in readily applicable recommendations for clinical practice for selection of high-risk patients for ECG screening and monitoring. ECG screening and monitoring is recommended before and following the start of QTc-prolonging antidepressants in the presence of vulnerability to QTc prolongation or two or more risk factors (age $>65$ years, female sex, concomitant use of a QTc-prolonging drug or concomitant use of a drug that influences the metabolism of a QTc-prolonging drug, cardiac disease, excessive dosing and specific electrolyte disturbances).

\section{Key Points}

Evidence from systematic reviews and metaanalyses with respect to risk factors for antidepressant-induced cardiac arrhythmia or sudden (cardiac) death is insufficient to generate relative risks for individual risk factors.

We present clinically applicable consensus guidelines for the selection of high-risk patients for electrocardiogram (ECG) screening and monitoring during antidepressant use.

ECG screening and monitoring is recommended before and following the start of QTc-prolonging antidepressants in the presence of known vulnerability to QTc prolongation or two or more risk factors (age $>65$ years, female sex, concomitant use of a QTc-prolonging drug or concomitant use of a drug that influences the metabolism of a QTcprolonging drug, cardiac disease, excessive dosing and specific electrolyte disturbances).

\section{Introduction}

The effect of psychotropic drugs on cardiac repolarization has increasingly gained attention in research and clinical practice over the last 2 decades. The absolute risk of cardiac arrhythmia, such as Torsade de Pointes (TdP), is generally low [14 per 10,000 patients over 1 year $(95 \%$ confidence interval 11-17/10,000)], and sudden cardiac

16 Department of Psychiatry, Warneford Hospital, University of Oxford, Oxford, UK

17 Department of Psychiatry, Radboudumc, Nijmegen, The Netherlands death as a consequence of all cardiac arrhythmias in general occurs even more rarely [1]. However, the tragic anecdotes of physically healthy patients encountering cardiac arrest and sudden (cardiac) death after the use of psychotropic drugs have underscored that some of these drugs may increase the risk of arrhythmias. This proarrhythmic effect is often marked by a prolongation of the QT interval/QTc interval (QT interval corrected for heart rate) on an electrocardiogram (ECG) [2].

Of the psychotropic drugs, antipsychotics are well known for their QT(c)-prolonging effects and association with TdP and sudden cardiac death, although the available evidence may not support this reputation per se [3]. The incidence rate of sudden cardiac death in users of antipsychotics was 2.9 per 1000 patient-years-a significantly doubled risk compared with (non-psychiatric) nonusers [3]. Some antidepressants have proven to also prolong the QT(c) interval. Although a recent meta-analysis showed significant QTc prolongation by tricyclic antidepressants (TCAs; doxepin, nortriptyline and amitriptyline) and some selective serotonin reuptake inhibitors (SSRIs; citalopram, escitalopram and sertraline) relative to placebo [4], CredibleMeds, the internationally renowned source for evidence-based classification of drugs according to their QTc-prolonging abilities, only classifies citalopram and escitalopram as antidepressant drugs with a known risk of $\mathrm{TdP}$, and clomipramine, desipramine, imipramine, nortriptyline, mirtazapine, trimipramine and venlafaxine as antidepressants with a possible risk of TdP [5]. Moreover, the US FDA has issued several drug safety communications, including for citalopram in 2011 and 2012. The warnings stated that citalopram use could lead to abnormal heart rhythms, and prescription doses should not exceed $40 \mathrm{mg} /$ day in adults and $20 \mathrm{mg}$ in patients $>65$ years of age $[6,7]$.

To make a proper estimation of the risk of arrhythmia, the combination of characteristics of the antidepressant therapy and patient-specific factors should be taken into account. A number of risk factors that add to the arrhythmia risk, including non-cardiac risk factors, have been proposed, including female sex, older age, (ischemic) heart disease or a history thereof, electrolyte disturbances (including hypomagnesemia, hypokalemia and hypocalcemia), pharmacokinetic and pharmacodynamic genetic factors, congenital long QTc syndrome, and a range of other medical conditions [8-11]. Several studies aimed to verify and synthesize the available evidence for QTc prolongation into a risk score for use in non-psychiatric hospitalized patients $[12,13]$. In addition, it has been shown that the risk for arrhythmia increases with increasing numbers of such risk factors [8-10]; however, the relative risks of the individual risk factors for the outcome arrhythmia are still unclear. 
Apart from the ECG monitoring recommendations in the Summary of Product Characteristics (SmPC) for individual QT(c)-prolonging antidepressant drugs, there are no clear and concrete national or international guidelines on if, when, and how often an ECG should be performed before and during treatment with antidepressant drugs. In the Dutch multidisciplinary guideline for depression, only with TCA treatment in elderly patients is an ECG recommended before the start of treatment (although this is to rule out contraindications such as a right bundle branch block to assist in drug choice, which is not the focus of this consensus document) and ECG monitoring with nortriptyline treatment in elderly with cardiac risk [14]. Although some of the available international depression guidelines do not mention ECG monitoring with antidepressant treatment $[15,16]$, other depression guidelines and some consensus documents for the management of QT(c) prolongation emphasize that special attention should be given to 'highrisk' patients in order to prevent unfavorable cardiac outcomes [8, 10, 17-19]. For example, in a consensus document, Dodd and colleagues suggest ECG monitoring during the use of TCAs [20]. They also recommend considering ECG monitoring during SSRI and serotonin noradrenaline reuptake inhibitor (SNRI) treatment in 'highrisk' individuals, although they consider it usually unnecessary [20]. However, how 'high-risk' must be quantified in clinical practice remains an enigma that hampers implementation of such recommendations in clinical practice.

Therefore, we aimed to answer the following important, unanswered questions in order to prevent cardiac arrhythmia and sudden (cardiac) death during antidepressant use: (1) should the ECG be monitored with antidepressant treatment, and (2) if so, for which patients (with which risk factors), when, and how often?

\section{Methods}

\subsection{The Multidisciplinary Expert Panel}

In 2015, the Dutch Network for Quality Development in Mental Health Care funded the development of recommendations about the prevention, monitoring, and treatment of side effects of psychotropic drugs. A multidisciplinary expert panel for antidepressant drugs addressed the association between the use of antidepressants and proarrhythmic effects and related ECG monitoring issues. The expert panel for antidepressants consisted of four psychiatrists (two being specialized in the treatment of children/adolescents or elderly patients, one additionally in training as a clinical pharmacologist), a general practitioner, an internist clinical pharmacologist, a nursing specialist, a patient representative, three (hospital) pharmacists, and a postdoctoral researcher.

\subsection{Literature Search}

In order to retrieve relevant literature as a base for our recommendations, two authors (AS and MS) searched for studies addressing the relative risks of risk factors (such as older age and female sex) for cardiac arrhythmia and sudden (cardiac) death, associated with antidepressant use. We did not use a restriction in the time period of publication and applied no language restrictions. For practical reasons and because of time restrictions for the project, we limited our search to systematic reviews and metaanalyses.

We conducted a search of MEDLINE using the search strategy "Psychotropic Drugs"[MeSH] AND ((()Arrhythmias, Cardiac[MeSH] OR arrhythmi*[tiab] OR proarrhythmi*[tiab] OR long QT[tiab] OR (prolong*[tiab] AND QT[tiab]) OR torsade de pointes[tiab] OR torsades de pointes[tiab] OR Death, Sudden, Cardiac[MeSH] OR cardiac death[tiab] OR cardiac mortality[tiab] OR "Cardiovascular Diseases/mortality" $[\mathrm{MeSH}]$ ) AND (druginduced[tiab] OR drug effects[sh] OR adverse effects[sh])) AND (Risk factors[MeSH] OR risk factors[tiab] OR prognost*[tiab] OR predict*[tiab])) AND (systematic*[tiab] OR review*[tiab] OR meta-analysis[tiab] OR MetaAnalysis[ptyp] OR systematic[sb])). We used the broader term 'psychotropic drugs' instead of antidepressant-specific search terms because a pilot search showed insufficient studies on risk factors for cardiac arrhythmia and sudden (cardiac) death when we used antidepressants in the search. Bibliographies of retrieved studies were scanned for additional systematic reviews and meta-analyses. We performed the last update of the search on 14 March 2017.

\subsection{Selection Criteria}

The study selection was performed independently by two authors (AS and MS) and discrepancies were resolved through discussion. We selected systematic reviews and meta-analyses of studies in (older) adult patients using psychotropic drugs registered in The Netherlands. Papers limited to overdose, intoxication, supratherapeutic dosage or antidepressants as add-on intervention were excluded. In our selection, we focused on cardiac arrhythmia and sudden (cardiac) death as outcomes and excluded publications solely on QTc interval as a surrogate measure for cardiac arrhythmia and sudden (cardiac) death. The latter was applied because QTc intervals are of limited value due to the use of different formulas to correct for heart rate (e.g. Bazett or Fridericia) and because the relationship between drug-induced QTc prolongation and the likelihood of 
arrhythmia appears to be, at best, modest and neither linear nor straightforward [21, 22]. Eligible studies should report on the risk for cardiac arrhythmia and sudden (cardiac) death in association with risk factors [e.g. age, sex, cardiac disease, electrolyte disturbances, prolonged QT(c) interval (syndrome), use of QT(c)-prolonging drugs].

\subsection{Formulation of Recommendations}

In order to formulate recommendations, the multidisciplinary expert panel received a summary of extracted data from selected studies. The odds ratios, relative risks and/or prevalences of cardiac arrhythmia and sudden (cardiac) death associated with the investigated risk factors were presented for each review/meta-analysis. This evidence served as input for discussion on the indication and timing for ECG screening and monitoring at treatment initiation and during use of antidepressants. When consensus was reached, each recommendation was graded evidence level 1-4 according to the Dutch criteria for evidence-based guideline development (EBRO), based on the Appraisal of Guidelines Research and Evaluation (AGREE) Collaboration [23-25].

\section{Results of the Literature Research}

We retrieved 100 publications of potential systematic reviews and/or meta-analyses (see electronic supplementary material). Seven titles and abstracts appeared to match our inclusion criteria; all seven studies were in English. Based on their full-text, four publications met our selection criteria [26-29]. The major reasons for exclusion of publications were failure to meet our population criterion, no investigation of risk factors, or solely outcomes other than cardiac arrhythmia and sudden (cardiac) death.

In a systematic review of TdP cases, Meyer-Massetti et al. investigated the prevalences of a predefined set of risk factors (age, sex, dose, electrolyte imbalance, cardiac disease, concomitant proarrhythmic drugs and other drugs influencing cardiac function and baseline QTc) in 54 patients with intravenous administration of the antipsychotic haloperidol [27]. Vieweg et al. provided prevalences of various risk factors in only a small number of TdP cases ( $n=4)$ associated with the antipsychotic risperidone [28]. Because of the low number of cases and the focus on antipsychotics, the results of these studies were not generalizable to patients using antidepressants and these two systematic reviews were therefore excluded.

Zeltser et al. performed a systematic review of TdP cases and described the prevalence of six risk factors for arrhythmia in 249 cases of TdP induced by non-cardiac drugs [29]. In brief, 70 of these cases (28.1\%) were caused by psychotropic drugs, mainly, but not exclusively, by antipsychotics (not further specified). Of the psychotropic drug-induced TdP cases, $71.4 \%$ were female, $44.7 \%$ used additional drugs that caused drug interactions (i.e. impairs the metabolism of QT-prolonging drugs or concomitant use of two or more QT-prolonging drugs), 43.1\% had existing cardiac disease, $27 \%$ used an excessive dose (leading to drug toxicity but excluding cases of suicidal overdose), $17.9 \%$ had hypokalemia, and $17.1 \%$ had a vulnerability to QT prolongation (familial history of long QT syndrome, history of drug-induced TdP, prolonged QT interval before drug administration). Cases using psychotropic drugs had, on average, 2.2 risk factors. No odds ratios for the different risk factors were given.

A study by Åström-Lilja et al. investigated the prevalence of a small set of risk factors for arrhythmia in druginduced TdP [26]. Since Zeltser et al. did not assess age as a risk factor, the study by Aström-Lilja et al. was considered of substantial added value for the expert panel, despite the fact that it was not a systematic review or meta-analysis. This series of 88 cases was based on data from the Swedish pharmacovigilance database, which was not restricted to psychotropic drugs [8/88 (9\%) used antidepressants]. The age of the TdP cases ranged from 15 to 90 years, with the median age being 74 years. Seventy-two percent of the TdP cases were over 65 years of age. Existing heart disease, female sex, and hypokalemia were present in 90,70 , and $12 \%$ of cases, respectively, while two or more established risk factors were present in $85 \%$ of cases $(75 / 88)$.

\section{Considerations of the Expert Panel}

In order to translate the available evidence to recommendations for daily clinical practice, we addresses several issues.

First, the expert panel concluded that the relative contributions of risk factors to cardiac arrhythmias and sudden (cardiac) death during antidepressant use could not be determined since no systematic reviews or meta-analyses addressed this topic specifically.

Second, the absence of a reference group (e.g. drug-free TdP cases or patients using psychotropic drugs who did not develop TdP) in the included reports hampered us to put the prevalences of risk factors into perspective and/or calculate odds ratios or relative risks. Therefore, it is impossible to draw firm conclusions on the relative importance of certain factors or the definition of a high-risk population for ECG screening and monitoring.

Third, we took the average time to steady state into account when drafting the advice about the timing of the follow-up ECG after reaching the target dose of treatment 
with a QTc-prolonging antidepressant. Time to steady state is four to five times the half-life of the drug, which is approximately 1 week for antidepressant drugs.

Fourth, the expert panel would like to point out that the current literature is inconclusive regarding the intraindividual circadian variation in the length of the QTc interval, which may range from $<10 \mathrm{~ms}$ to up to $75-100 \mathrm{~ms}$ $[21,30,31]$. It is therefore preferable to register ECG recordings at fixed time points during the day in order to avoid bias in the change between two subsequent ECGs as a result of circadian variation.

Fifth, because threshold values for a prolonged QTc interval, such as those of the AHA/ACCF/HRS, are the result of consensus, the expert panel suggested to consider a similar threshold value for women and men (i.e. $>450 \mathrm{~ms}$, instead of $>450 \mathrm{~ms}$ for men and $>460 \mathrm{~ms}$ for women [32]), which will reduce complexity for clinical practice. This is supported by the consideration that women are at increased risk for QTc prolongation and TdP; lowering the threshold for a prolonged QTc interval to $>450 \mathrm{~ms}$ in women also, will result in earlier identification than with $>460 \mathrm{~ms}$ [33].

Last, the expert panel noted that because of the actions of sex hormones on the QTc interval, the difference between women and men in the prolonged QTc-interval risk disappears during menopause [34, 35].

\section{Recommendations}

Based on the limited literature results and the consensus reached by our expert panel, we formulated the following recommendations, all graded 4-expert opinion (Fig. 1) [23-25].

- ECG screening and monitoring is recommended for antidepressants with known or possible risk of TdP according to CredibleMeds (citalopram, clomipramine, desipramine, escitalopram, imipramine, mirtazapine, nortriptyline, trimipramine and venlafaxine) [5]) if the patient

1. is having a known risk of QTc prolongation:

- known prolonged QTc interval;

- history of TdP;

- family history of long QTc syndrome or sudden cardiac death; and choosing an antidepressant without QTc-prolonging ability is not possible; Remark: If the risk of QTc prolongation cannot be determined, it can be considered absent for decision-making purposes.

or
2. has two or more of the risk factors listed below (based on the two reviews from the literature search discussed above [26, 29]):

- age over 65 years;

- female sex;

- concomitant use of a QTc-prolonging drug (list of drugs with a known risk of TdP according to CredibleMeds [5]) or concomitant use of a drug that influences the metabolism of the antidepressant with known or possible risk of TdP (i.e. citalopram, clomipramine, desipramine, escitalopram, imipramine, mirtazapine, nortriptyline, trimipramine and venlafaxine [5]);

- cardiac disease (myocardial infarction, heart failure, valvulopathy, cardiomyopathy);

- excessive dosing (higher than the highest dose according to the SmPC, or standard dose with a relevant kidney or liver problem);

- specific electrolyte disturbances (hypocalcemia, hypokalemia, hypomagnesemia) [26, 29].

Additional remark: In case of a strong suspicion of electrolyte disturbances, e.g. with alcoholism, anorexia nervosa, diarrhea, the use of loop diuretics, etc., the calcium, potassium and magnesium serum level should be quantified.

- Paroxetine, duloxetine and fluoxetine do not affect the QTc interval in comparison with placebo, while fluvoxamine shortens the QTc interval compared with placebo [4]. For other antidepressants, evidence for determining their QTc-prolonging ability is currently insufficient.

- In general, it is unnecessary to make an ECG prior to starting any antidepressant if the patient is not at risk of QTc prolongation, and all of the abovementioned risk factors for cardiac arrhythmia or conduction disorders are absent.

- ECG screening and monitoring should consist of an ECG before treatment initiation and 1 week after reaching the target dose of a the QTc-prolonging antidepressant (i.e. when steady state has been reached).

- In case the ECG shows a QTc interval of $450 \mathrm{~ms}$ or above, it is advisable to consult a cardiologist.

Remark: although we focus on the QTc interval in this consensus document, the PQ and QRS intervals may also be relevant during psychotropic drug use.

- If needed, for example in acute situations or highly severe mentally ill cases, the treating physician can 
Fig. 1 Decision tree for ECG monitoring with antidepressant treatment. QTC QT interval corrected for heart rate, ECG electrocardiogram, $T d P$ Torsades de Pointes. ${ }^{\text {a}}$ Based on CredibleMeds.org [5], which represents available and evolving evidence that is constantly re-evaluated when new evidence becomes available. ${ }^{\mathrm{b}}$ If the risk of QTc prolongation cannot be determined, it can be considered absent for decision-making purposes. ${ }^{\mathrm{C}}$ In case of a strong suspicion of electrolyte disturbances, e.g. with alcoholism, anorexia nervosa, diarrhea, use of loop diuretics, etc., the calcium, potassium and magnesium serum level should be quantified

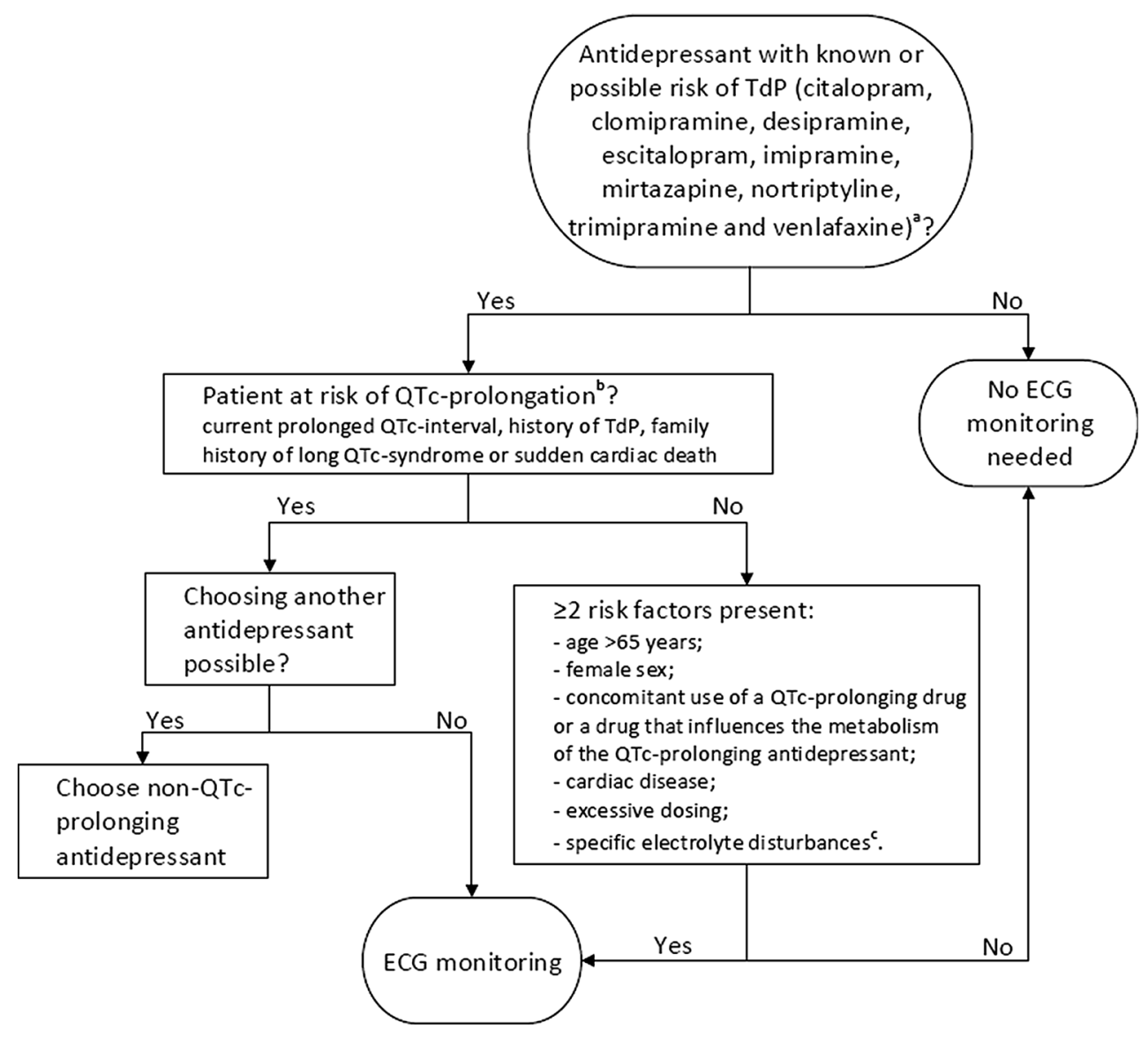

deviate from the recommended ECG at treatment initiation.

\section{Discussion}

In this Dutch consensus document on ECG screening and monitoring in patients using antidepressants, no need for ECG screening and monitoring is recommended in patients without QTc-prolongation vulnerability and other risk factors for cardiac arrhythmia during antidepressant therapy. ECG screening and monitoring is recommended before and following the start of antidepressants with known or possible risk of TdP (i.e. citalopram, clomipramine, desipramine, escitalopram, imipramine, mirtazapine, nortriptyline, trimipramine and venlafaxine [5]) in the presence of vulnerability to QTc prolongation or two or more risk factors (age $>65$ years, female sex, concomitant use of a QTc-prolonging drug or concomitant use of a drug that influences the metabolism of the QTc-prolonging antidepressant, cardiac disease, excessive dosing and specific electrolyte disturbances).

Some authors, guidelines, and drug labels state that all patients receiving QTc-prolonging psychotropic medication should be monitored, but most experts and authors of previous reviews emphasize that ECG screening and monitoring is only necessary in high-risk patients $[20,22,36]$. Current guidelines do not provide uniform recommendations with respect to ECG screening and monitoring in patients using antidepressants. Unfortunately, there is a lack of evidence that ECG screening and monitoring indeed can prevent cases of arrhythmia or sudden cardiac death. For this, the 'number needed to ECG' (NNE) would be an interesting number to indicate the number of patients who should be monitored (with consecutive ECGs) to prevent one additional death or adverse event due to QTc prolongation. Given the rising costs of (mental) health, the apparent pressure to increase productivity, and high administrative workload, it would also be appropriate to perform a proper health economical cost-benefit assessment before issuing general recommendations on ECG screening and monitoring. An optimized balance between costs and yield of ECG screening and monitoring to detect aberrances would support clinicians in their treatment decisions, while unnecessary ECGs could be eliminated as much as possible.

Because of a relative lack of systematic reviews and meta-analyses that compare risk factors (despite a number 
of studies on risk scores for QTc prolongation), the relative risks of risk factors for cardiac arrhythmia and sudden (cardiac) death cannot be established/quantified, which hampers a better determination of a 'high-risk' population. Therefore, we decided to consider the various risk factors that were put forward in two earlier reviews of TdP cases $[26,29]$. The contribution of these risk factors to cardiac arrhythmia and sudden (cardiac) death have not yet been quantified. However, the results of our literature study are corroborated by a recent systematic review by Vandael et al. of large randomized controlled trials and observational studies that assessed the level of evidence for several factors to increase the risk of QTc prolongation in a general population [37]. Although strong evidence was found for a few risk factors (including hypokalemia and use of drugs with known risk of TdP as listed by CredibleMeds [5]), little or no evidence was found for many other risk factors [37].

Based on the identified risk factors, Vandael et al. subsequently aimed at developing a risk score to identify patients at low/high risk for QTc prolongation in an observational study in hospital patients with a first prescription for haloperidol or a QTc-prolonging antibiotic or antimicotic [38]. The RISQ-PATH index, using more and some other risk factors than we mention in this paper, was able to exclude low-risk patients from further ECG followup when starting QTc-prolonging drugs [high sensitivity (96.2\%)], but also resulted in many false positives [low specificity (32.9\%)] [38]. Because the aim of a risk score would be to safely exclude patients with low risk from the total population, this tool seems a promising instrument. The RISQ-PATH index is similar to our recommendations for ECG screening and monitoring, except that it additionally contains a weighing of risk factors based on the level of evidence-not relative risk-found in their systematic review. Instead of a risk score, we chose the dichotomous cut-off of two risk factors as a threshold, based on the evidence of a higher risk of cardiac arrhythmia outcomes with an increasing number of risk factors, the average number of risk factors found in the two reviews from our literature study, the general low absolute risk of our primary outcomes, and approximation of the same high sensitivity (i.e. yield of 'high risk' cases for cardiac arrhythmia and/or sudden [cardiac] death vs. costs and logistics of obtaining ECGs in mental health). Further research is warranted to quantify the relative risks of each risk factor and the sensitivity/specificity of a risk score in people prescribed an antidepressant before implementation of such a weighted risk index in clinical practice. To obtain efficacy and (cost-)effectiveness data, ideally a randomized controlled trial is performed to assign patients to be monitored according to their risk score versus treatment as usual. This would also enable to determine an NNE to guide future ECG screening and monitoring guidelines. However, given the low prevalence of QTc prolongation and outcomes such as cardiac arrhythmia and sudden death, performing such an RCT requires large numbers of patients and resources. Therefore a non-randomized approach as used by Vandael et al. might be most feasible [38].

For implementation, the counting of risk factors to determine the need for ECG screening and monitoring would ideally be incorporated in automated clinical decision support systems that alert prescribers to obtain ECG screening and monitoring [39]. The combination of information from electronic medical records and an electronic prescribing system would be supportive in preventing undesirable outcomes of QTc-prolonging drugs and increased efficacy of ECG screening and monitoring in specifically high-risk patients [39].

\subsection{Strength and Limitations}

The strengths of this paper are the clear recommendations to select 'high-risk patients' for ECG screening and monitoring when prescribing a QTc-prolonging antidepressant, which are easily applicable in clinical practice. However, some critical issues must be addressed.

The first limitation of our work is the restriction of our search to systematic reviews and meta-analyses, which provided a summary of available case-series but did not exclude the possibility that reports such as additional caseseries or cohort studies might have been missed. Furthermore, we could only identify papers on patients using psychotropic drugs in general, without a focus on antidepressants. We loosened our restriction to include reports on antidepressants only after our pilot search showed insufficient studies when we used 'antidepressants' as a search term. Given our restriction of systematic reviews and metaanalyses, we think this was the best compromise to retrieve reviews on patients using antidepressants and risk factors for cardiac arrhythmia. From four studies initially selected, only two studies were informative as they included a larger number of patients also using antidepressants. One of these was not a systematic review, but was still considered relevant in the absence of additional adequate evidence from systematic reviews and meta-analyses. Our recommendations are based on the currently limited available literature and almost entirely on expert opinion (specific for antidepressants) to make them readily applicable in clinical practice.

Second, risk factors for unfavorable cardiac (arrhythmia) outcomes may be generalizable across populations using psychotropic and other drugs. If so, a broader review of studies in patients using non-psychotropic drugs might have revealed more information, e.g. Vandael et al. [37]. We suggest to evaluate and amend our recommendations 
after new relevant studies have been released, with additional practice-based experience following implementation in clinical practice.

Third, we did not include a recommendation on the need for periodic follow-up (e.g. yearly) after the baseline and follow-up ECG during antidepressant use. Because evidence on the timing of cardiac arrhythmia and sudden (cardiac) death relative to the start of antidepressant therapy is scarce, and existence of risk factors may vary with time, it cannot be expected that a normal ECG after treatment initiation will indefinitely predict a low risk of later cardiac arrhythmia outcomes. Therefore, it might be necessary to repeat ECG screening and monitoring over time, for which additional recommendations must be formulated when more data are available.

Fourth, the evidence for the QTc-prolonging abilities of psychotropic drugs is scarce, with inconsistent results. For sertraline, for example, results on QTc prolongation are contradictory [40, 41]. The initial classification based on a recent comparative systematic review and meta-analysis [4] was carefully re-evaluated. Despite this systematic review, and in the absence of more specific evidence for fatal cardiac events for the remaining antidepressants on the list from this review, we decided to follow the CredibleMeds classification for our recommendations. Together with this choice, we would like to emphasize again that our recommendations are not final and may change when additional evidence appears.

Finally, ECG screening and monitoring recommendations should be actively and adequately implemented. Previous research has shown that the introduction of new guidelines, consensus statements, or (national) quality improvement programs alone has been minimally effective in improving screening and monitoring rates [42-45]. Specifically, compliance with these recommendations for the risk management of QTc prolongation by antidepressants may be poor [46-48].

\section{Conclusions}

We present specific consensus recommendations for ECG screening and monitoring in patients using antidepressants. Although these recommendations are based on limited evidence in the currently scarcely available literature, the elaboration of the available evidence in combination with clinical expertise and the multidisciplinary consensus process resulted in readily applicable recommendations, which, as a next step, need to be empirically validated. Future research should evaluate these recommendations, ideally in an RCT comparing their implementation with care as usual, combined with a health technology assessment, to assess the NNE and cost-benefit ratio. The recommendations should thereafter be evaluated and amended if necessary.

Funding This project was funded by the Dutch Network for Quality Development in Mental Health Care (Grants P140019 and P140040 to Henricus Ruhé and Roberto Bakker). This organization had no influence on the content of this study/manuscript.

\section{Compliance with Ethical Standards}

Conflict of interest Henricus Ruhé obtained an investigator-initiated trial grant and speaking fees from Lundbeck BV, which are not related to the current work. Mirjam Simoons, Adrie Seldenrijk, Hans Mulder, Tom Birkenhäger, Mascha Groothedde-Kuyvenhoven, Rob Kok, Cornelis Kramers, Wim Verbeeck, Mirjam Westra, Eric van Roon and Roberto Bakker have no conflicts of interest that are directly relevant to the content of this study.

Ethical approval Ethical approval was not applicable for this study.

Patient consent Patient consent was not applicable for this study.

Open Access This article is distributed under the terms of the Creative Commons Attribution-NonCommercial 4.0 International License (http://creativecommons.org/licenses/by-nc/4.0/), which permits any noncommercial use, distribution, and reproduction in any medium, provided you give appropriate credit to the original author(s) and the source, provide a link to the Creative Commons license, and indicate if changes were made.

\section{References}

1. Coupland C, Hill T, Morriss R, Moore M, Arthur A, HippisleyCox J. Antidepressant use and risk of cardiovascular outcomes in people aged 20-64: cohort study using primary care database. BMJ. 2016;352:i1350.

2. Frommeyer G, Eckardt L. Drug-induced proarrhythmia: risk factors and electrophysiological mechanisms. Nat Rev Cardiol. 2016;13:36-47.

3. Ray WA, Chung CP, Murray KT, Hall K, Stein CM. Atypical antipsychotic drugs and the risk of sudden cardiac death. N Engl J Med. 2009;360:225-35.

4. Beach SR, Kostis WJ, Celano CM, Januzzi JL, Ruskin JN, Noseworthy PA, et al. Meta-analysis of selective serotonin reuptake inhibitor-associated QTc prolongation. J Clin Psychiatry. 2014;75:e441-9.

5. CredibleMeds (AZCERT I). QTDrugs Lists. 2017. https:// crediblemeds.org/new-drug-list/. Accessed 29 Nov 2017.

6. US Food and Drug Administration. FDA Drug Safety Communication: Revised recommendations for Celexa (citalopram hydrobromide) related to a potential risk of abnormal heart rhythms with high doses. 2012. https://www.fda.gov/Drugs/ DrugSafety/ucm297391.htm. Accessed 28 Sept 2017.

7. US Food and Drug Administration. FDA Drug Safety Communication: Abnormal heart rhythms associated with high doses of Celexa (citalopram hydrobromide). 2011. https://www.fda.gov/ Drugs/DrugSafety/ucm269086.htm. Accessed 2 Mar 2017.

8. Drew BJ, Ackerman MJ, Funk M, Gibler WB, Kligfield P, Menon V, et al. Prevention of torsade de pointes in hospital settings: a scientific statement from the American Heart Association and the 
American College of Cardiology Foundation. J Am Coll Cardiol. 2010;55:934-47.

9. Roden DM. Drug-induced prolongation of the QT interval. N Engl J Med. 2004;350:1013-22.

10. Ames D, Camm J, Cook P, Falkai P, Gury C, Hurley R, et al. Minimizing the risks associated with QTc prolongation in people with schizophrenia. A consensus statement by the Cardiac Safety in Schizophrenia Group. Encephale. 2002;28:552-62.

11. Niemeijer MN, van den Berg ME, Eijgelsheim M, Rijnbeek PR, Stricker BH. Pharmacogenetics of drug-induced QT interval prolongation: an update. Drug Saf. 2015;38:855-67.

12. Anderson HN, Bos JM, Haugaa KH, Morlan BW, Tarrell RF, Caraballo PJ, et al. Prevalence and outcome of high-risk QT prolongation recorded in the Emergency Department from an Institution-Wide QT Alert System. J Emerg Med. 2018;54:8-157.

13. Tisdale JE, Jaynes HA, Kingery JR, Mourad NA, Trujillo TN, Overholser BR, et al. Development and validation of a risk score to predict QT interval prolongation in hospitalized patients. Circ Cardiovasc Qual Outcomes. 2013;6:479-87.

14. Spijker J, Bockting CLH, Meeuwissen JAC, Vliet van IM, Emmelkamp PMG, Hermens MLM et al. Multidisciplinary Guideline Depression (third revision). 2013. https://www. ggzrichtlijnen.nl/depressie. Accessed 28 Sept 2017. Article in Dutch.

15. National Institute for Health and Care Excellence (NICE). Depression in adults: recognition and management (CG 90). 2009. https://www.nice.org.uk/guidance/cg90/resources/depressionin-adults-recognition-and-management-pdf-975742636741. Accessed 28 Sept 2017.

16. Kennedy SH, Lam RW, McIntyre RS, Tourjman SV, Bhat V, Blier P, et al. Canadian Network for Mood and Anxiety Treatments (CANMAT) 2016 clinical guidelines for the management of adults with major depressive disorder: section 3. Pharmacological treatments. Can J Psychiatry. 2016;61:540-60.

17. Royal College of Psychiatrists. CR190: Consensus statement on high-dose antipsychotic medication. 2014. http://www.rcpsych. ac.uk/files/pdfversion/CR190.pdf. Accessed 28 Sept 2017.

18. Cleare A, Pariante CM, Young AH, Anderson IM, Christmas D, Cowen PJ, et al. Evidence-based guidelines for treating depressive disorders with antidepressants: a revision of the 2008 British Association for Psychopharmacology guidelines. J Psychopharmacol. 2015;29:459-525.

19. American Psychiatric Association. Practice guideline for the treatment of patients with major depressive disorder. 2010. https://psychiatryonline.org/pb/assets/raw/sitewide/practice_guidelines/guidelines/mdd.pdf. Accessed 28 Nov 2017.

20. Dodd S, Malhi GS, Tiller J, Schweitzer I, Hickie I, Khoo JP, et al. A consensus statement for safety monitoring guidelines of treatments for major depressive disorder. Aust N Z J Psychiatry. 2011;45:712-25.

21. Beach SR, Celano CM, Noseworthy PA, Januzzi JL, Huffman JC. QTc prolongation, torsades de pointes, and psychotropic medications. Psychosomatics. 2013;54:1-13.

22. Shah AA, Aftab A, Coverdale J. QTc prolongation with antipsychotics: is routine ECG monitoring recommended? J Psychiatr Pract. 2014;20:196-206.

23. Quality institute for health care, CBO. Evidence-based guideline development (EBRO): Manual for working group members. 2007. www.ha-ring.nl/download/literatuur/EBRO_handl_totaal. pdf. Accessed 28 Sept 2017. Article in Dutch.

24. Burgers JS, van Everdingen JJ. Evidence-based guideline development in the Netherlands: the EBRO platform. Ned Tijdschr Geneeskd. 2004;148:2057-9 (Article in Dutch).

25. AGREE Collaboration. Development and validation of an international appraisal instrument for assessing the quality of clinical practice guidelines: the AGREE project. Qual Saf Health Care. 2003;12:18-23.

26. Aström-Lilja C, Odeberg JM, Ekman E, Hagg S. Drug-induced torsades de pointes: a review of the Swedish pharmacovigilance database. Pharmacoepidemiol Drug Saf. 2008;17:587-92.

27. Meyer-Massetti C, Cheng CM, Sharpe BA, Meier CR, Guglielmo BJ. The FDA extended warning for intravenous haloperidol and torsades de pointes: how should institutions respond? J Hosp Med. 2010;5:E8-16.

28. Vieweg WV, Hasnain M, Hancox JC, Baranchuk A, Digby GC, Kogut C, et al. Risperidone, QTc interval prolongation, and torsade de pointes: a systematic review of case reports. Psychopharmacology. 2013;228:515-24.

29. Zeltser D, Justo D, Halkin A, Prokhorov V, Heller K, Viskin S. Torsade de pointes due to noncardiac drugs: most patients have easily identifiable risk factors. Medicine (Baltimore). 2003;82:282-90.

30. Zhang J, Dang Q, Malik M. Baseline correction in parallel thorough QT studies. Drug Saf. 2013;36:441-53.

31. Smetana P, Batchvarov V, Hnatkova K, Camm AJ, Malik M. Circadian rhythm of the corrected QT interval: impact of different heart rate correction models. Pacing Clin Electrophysiol. 2003;26:383-6.

32. Rautaharju PM, Surawicz B, Gettes LS, Bailey JJ, Childers R, Deal BJ, et al. AHA/ACCF/HRS recommendations for the standardization and interpretation of the electrocardiogram: part IV: the ST segment, T and U waves, and the QT interval: a scientific statement from the American Heart Association Electrocardiography and Arrhythmias Committee, Council on Clinical Cardiology; the American College of Cardiology Foundation; and the Heart Rhythm Society: endorsed by the International Society for Computerized Electrocardiology. Circulation. 2009;119:e241-50.

33. European Medicines Agency. ICH Topic E 14 The Clinical Evaluation of QT/QTc Interval Prolongation and Proarrhythmic Potential for Non-Antiarrhythmic Drugs. 2005. http://www.ema. europa.eu/docs/en_GB/document_library/Scientific_guideline/ 2009/09/WC500002879.pdf. Accessed 28 Sept 2017.

34. Sedlak T, Shufelt C, Iribarren C, Merz CN. Sex hormones and the QT interval: a review. J Womens Health (Larchmt). 2012;21:933-41.

35. Kurokawa J, Furukawa T. Non-genomic action of sex steroid hormones and cardiac repolarization. Biol Pharm Bull. 2013;36:8-12.

36. Warnier MJ, Holtkamp FA, Rutten FH, Hoes AW, de Boer A, Mol PG, et al. Quality of drug label information on QT interval prolongation. Int J Risk Saf Med. 2014;26:89-98.

37. Vandael E, Vandenberk B, Vandenberghe J, Willems R, Foulon V. Risk factors for QTc-prolongation: systematic review of the evidence. Int J Clin Pharm. 2017;39:16-25.

38. Vandael E, Vandenberk B, Vandenberghe J, Spriet I, Willems R, Foulon V. Development of a risk scorefor QTc-prolongation: the RISQ-PATH study. Int J Clin Pharm. 2017;39:424-32.

39. Schwartz PJ, Woosley RL. Predicting the unpredictable: druginduced QT prolongation and Torsades de Pointes. J Am Coll Cardiol. 2016;67:1639-50.

40. Castro VM, Clements CC, Murphy SN, Gainer VS, Fava M, Weilburg JB, et al. QT interval and antidepressant use: a cross sectional study of electronic health records. BMJ. 2013;346:f288.

41. Fisch C, Knoebel SB. Electrographic findings in sertraline depression trials. Drug Investig. 1992;4:305-12.

42. Mitchell AJ, Delaffon V, Vancampfort D, Correll CU, De Hert M. Guideline concordant monitoring of metabolic risk in people treated with antipsychotic medication: systematic review and meta-analysis of screening practices. Psychol Med. 2012;42:125-47. 
43. Dhamane AD, Martin BC, Brixner DI, Hudson TJ, Said Q. Metabolic monitoring of patients prescribed second-generation antipsychotics. J Psychiatr Pract. 2013;19:360-74.

44. Paton C, Adroer R, Barnes TR. Monitoring lithium therapy: the impact of a quality improvement programme in the UK. Bipolar Disord. 2013;15:865-75.

45. Morrato EH, Druss B, Hartung DM, Valuck RJ, Allen R, Campagna E, et al. Metabolic testing rates in 3 state Medicaid programs after FDA warnings and ADA/APA recommendations for second-generation antipsychotic drugs. Arch Gen Psychiatry. 2010;67:17-24.

46. Cheung D, Wolfe B, Wald H, Cumbler E. Unsafe use of intravenous haloperidol: evaluation of recommendation-concordant care in hospitalized elderly adults. $\mathrm{J}$ Am Geriatr Soc. 2013;61:160-1.

47. Jardin CG, Putney D, Michaud S. Assessment of drug-induced torsade de pointes risk for hospitalized high-risk patients receiving QT-prolonging agents. Ann Pharmacother. 2014;48:196-202.

48. Warnier MJ, Rutten FH, Souverein PC, de Boer A, Hoes AW, De Bruin ML. Are ECG monitoring recommendations before prescription of QT-prolonging drugs applied in daily practice? The example of haloperidol. Pharmacoepidemiol Drug Saf. 2015;24:701-8. 